\title{
Pneumonia Detection Using Artificial Neural Networks and Transfer Learning Model
}

\author{
U.M.Prakash, Hitarth Pandey, Abhishek Suryavanshi, K.R.Gokul Anand
}

\begin{abstract}
Developing a system that helps in detecting pneumonia in chest $x$-ray images of lungs at a high accuracy. Firstly, a raw image is being preprocessed with the help of Otsu Thresholding and an equalizer. This helps in detecting pneumonia clouds and identifying the ratio of healthy lung region to the total region minimum. The above task is determined by importing the original chest $x$-ray images in the dataset and then calculating the ratio. The preprocessed data is then fed into Inception V3 model that accurately predicts the percentage of how much pneumonia is spread. This helps in identifying pneumonia present in that area and helps determining the prescribed drugs to help them clear off the symptoms.
\end{abstract}

Index Terms: Otsu thresholding, Transfer learning, inception v3, white clouds, CNN

\section{INTRODUCTION}

Pneumonia is an infection which affects the lungs by affecting the air sacs present in it. Pneumonia causes inflammation in the air sacs, which makes the person difficult to breathe. Surveys conducted show us that pneumonia can be detected using chest $\mathrm{X}$ ray images. But in order to get insights from the CXR image, it requires various background knowledge. Accuracy of prediction comes from the experience that the person has in that field. This is where machine feed algorithm can help in predicting the results more accurately and classifying which kind of pneumonia it is.

The primary working of our algorithm is to first get the raw CXR image and then we preprocess the raw CXR image to get useful parameters. After that, this preprocessed image is fed to a pertained model which helps in determining the percentage in pneumonia.

The neural net algorithm which we use is InceptionV3. This is the main algorithm which helps in image classification. It takes an input image, process it and classify it under certain categories in this case pneumonia or not pneumonia. The given image is seen as an array of pixels by the algorithm. Based on the image resolution, it divides the image as height, width and dimension. Also, by the help of transfer learning we can determine various other factors and predict the outcome with accuracy.

There are various ways to conduct pneumonia detection on a machine level. The most significant level which is seen is with the help of transfer learning. Hence, it is very important to make use of transfer learning to understand more about the pneumonia behavior.

Revised Manuscript Received on July 05, 2019.

U.M.Prakash, CSE, SRM IST, CHENNAI, INDIA.

Hitarth Pandey, CSE, SRM IST, CHENNAI, INDIA

Abhishek Suryavanshi, CSE, SRM IST, CHENNAI, INDIA.

\section{DATASET AND EXPLORATION}

The dataset consists of 5216 train CXR images and 624 test CXR images with a validation folder for reference of 16 images classified as two folders Normal and Pneumonia. So, all over 6000 images are trained and tested to get the accurate results.

The dataset is first explored with the help of regression model to get a clear idea about how the images are scattered. The plot is shown below:

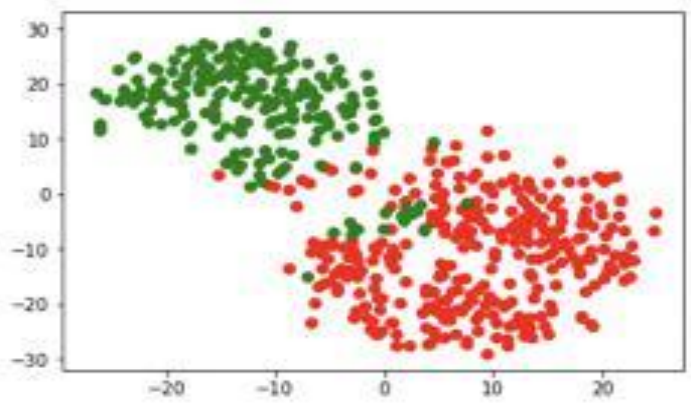

Figure 1: Normal and Pneumonia

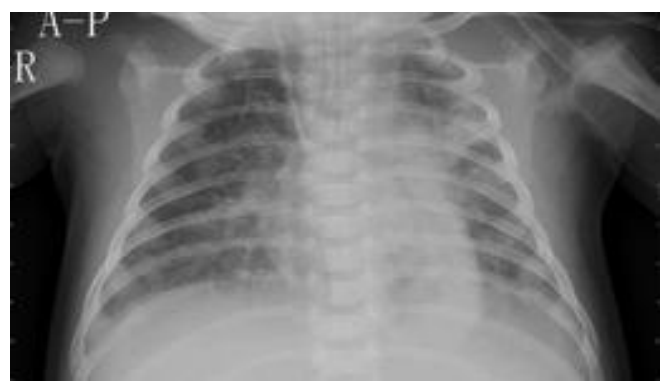

Figure 2: No Pneumonia

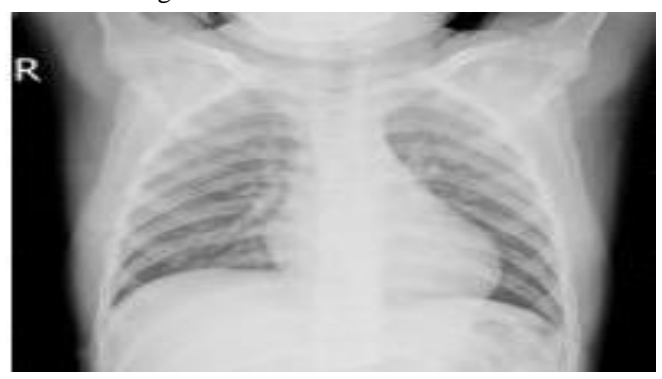

Figure 3: Pneumonia

The above plot is generated with the help of regression modeling which gives a clear idea about the only two possible classifications: The above pictures of CXR images are validated and hence the result is obtained. This gives us a

K.R.Gokul Anand, CSE, SRM IST, Published By: Blue Eyes Intelligence Engineering \& Sciences Publication 
clear idea of how the images have differences and the preprocessing is done easily.

\section{PROPOSED SYSTEM}

Here the approach is a combined set of different approaches that has been adopted recently to get a definite model and include an integration of all the other systems provided. The following steps are taken into considerations:

\section{A. Data Preprocessing}

As soon as the dataset is given the first step is to get a preprocessed image ready for training. This is achieved by some of the previous techniques that are Otsu threshold algorithm which creates a much denser image and also with the help of equalizer various other parts of the region are image. The below diagram shows how the preprocessing is achieved:

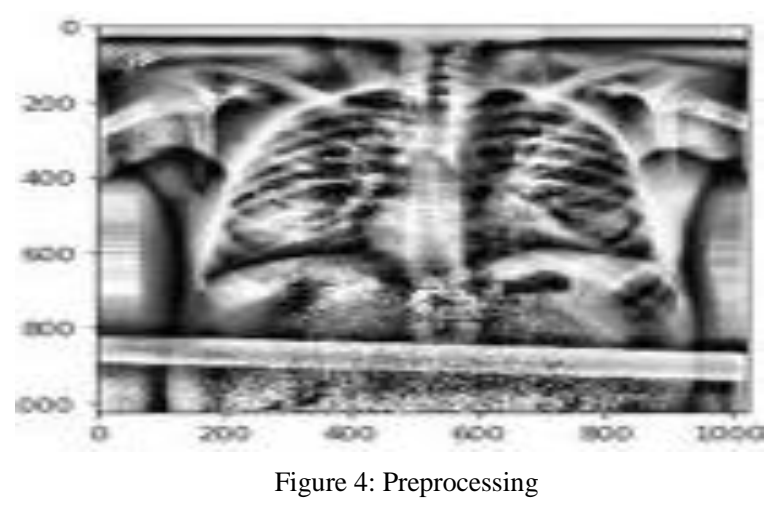

Here, the image has been sharpened multiple times to get the white clouds present inside the lungs.

\section{B. Model Building}

After running the preprocessed data, the next part is model building. This can be done with the help of various algorithms. Here, only two of those algorithms are used and the models are built

The two algorithms are:

- CNN (Convolution Neural Network)

- Transfer Learning (Inception V3)

These models are used to generate a rightful accurate results and prediction of the pneumonia to the normal region. What the two algorithms do is to get the classification done on the basis of the required preprocessed data. This is done in a quick manner and due to the previous preprocessed data being used, the output is quite accurate.

The confusion matrix and the accuracy graphs are shown:

The result shown below relates how we are close in case of the train and test data. The CNN Model offers $80 \%$ of accuracy while the Inception Model offers with $90 \%$.

The graph shown below is the reason why transfer learning is better than other machine learning models. This is due to the performance and a head start given to the transfer learning models. shown which helps in an in-depth knowledge about the CXR
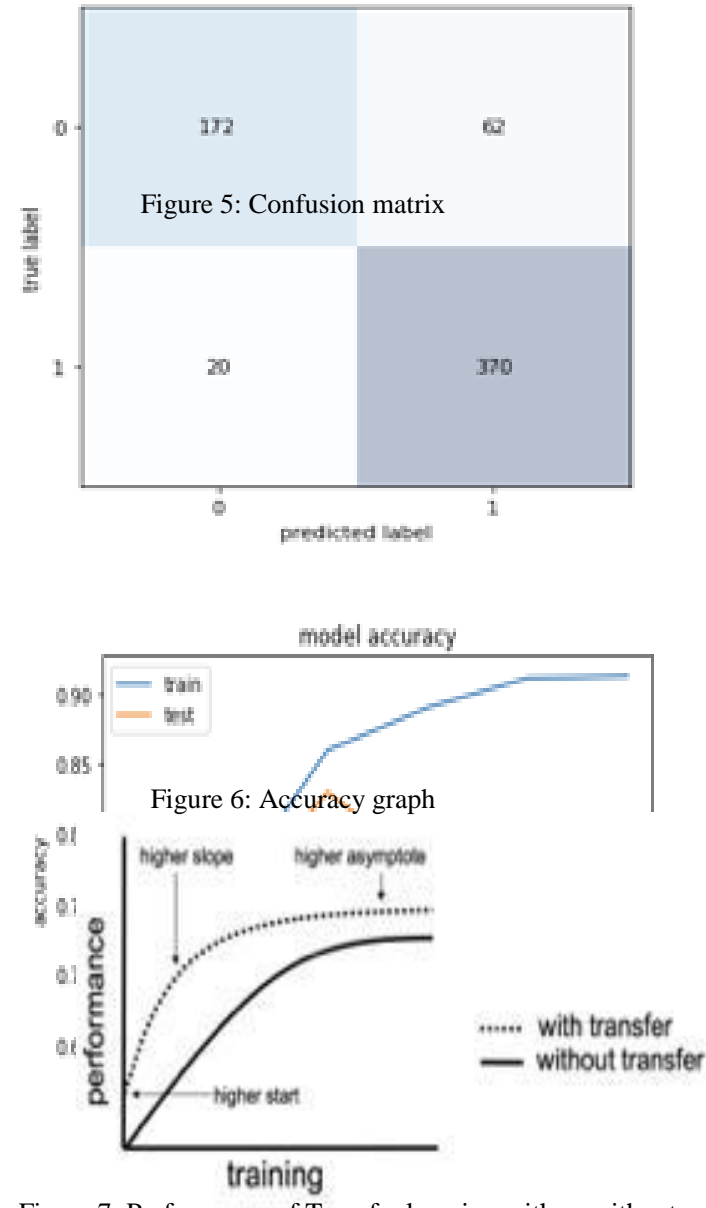

Figure 7: Performance of Transfer learning with or without

\section{Evaluation and Future Insights}

The final part is to evaluate the result and visualize the data. This is done because of getting the future insights.

The various future insights are listed below:

- The effect on the preprocessed image reduces the amount of job to train the model. Hence, the performance is increased.

- Higher performance due to pre trained models and preprocessed images.

- Obtaining accurate results in short amount of time.

- Using a combined machine learning algorithm to obtain dynamic results and hence generating various new techniques to solve the problem.

- Using the output or trained model for various medical facilities to obtain a performed task and help in the future world.

- Developing various coping mechanisms that will help detect pneumonia and diagnose to a certain extent which will help cure the disease.

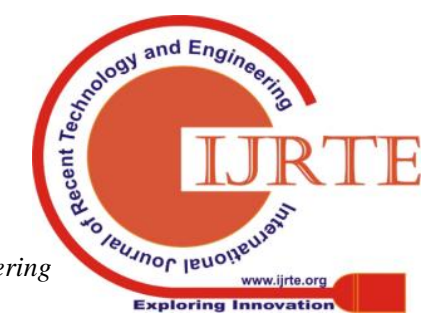




\section{CONCLUSION}

Pneumonia plays a very vital role in life of children. More than $20 \%$ of children are suffering from pneumonia according to WHO reports. Moreover, the early diagnosis helps in preventing the death. It does not diminish the effect.

The model provides an in-depth knowledge of curing the pneumonia at its best. This could also help us in other technological aspects where heath plays an important role in our daily lives. The important aspect is how to solve the problem with better performance and hence we provide that by the use of transfer learning.

\section{CONFLICT OF INTEREST}

The authors declare no conflict of interest.

\section{ACKNOWLEDGMENT}

We would like to thank SRM IST for providing us with a greater exposure and also for various research and surveys. Also, we would like to thank our guide U.M. Prakash for helping us getting to know about how to demonstrate and dictate to papers and also the procedure to gain more insights.

\section{REFERENCES}

1. Abhishek Sharma, Daniel Raju, Sutapa Ranjan "Detection of Pneumonia clouds in Chest X-ray using Image processing approach"-2017.

2. Cisneros-Velarde, P., Correa, M., Mayta, H., Anticona, C., Pajuelo, M., Oberhelman, R., Castaneda, B. (2016). Automatic pneumonia detection based on ultrasound video analysis. 2016 38th Annual International Conference of the IEEE Engineering in Medicine and Biology Society (EMBC). -2016.

3. Pranav Rajpurkar,Jeremy Irvin, Kaylie Zhu,Brandon Yang, Hershel Mehta, Tony Duan, Daisy Ding, Andrew Y Ng "CheXNet: Radiologist- Level Pneumonia Detection on Chest X-Rays with Deep Learning"- December 2017.

4. Pranav Rajpurkar, Jeremy Irvin, et al. "CheXNet: Radiologist-Level Pneumonia Detection on Chest X- Rays with Deep Learning”, 2017.

5. Diederik P. Kingma, Jimmy Lei Ba. Adam: "A Method for Stochastic Optimization",

6. S. Hwang, H.-E. Kim, J. Jeong, and H.-J. Kim, “A novel approach for tuberculosis screening based on deep convolution neural networks," in SPIE Medical Imaging. International Society for Optics and Photonics, 2016, pp. 97852W-97 852W.

7. A. Kumar, Y.-Y. Wang, K.-C. Liu, I.-C. Tsai, C.- C. Huang, and N. Hung, "Distinguishing normal and pulmonary edema chest $\mathrm{X}$-ray using gabor filter and svm," in Bioelectronics and Bioinformatics (ISBB), 2014 IEEE International Symposium on. IEEE, 2014, pp. 1-4.

8. Campadelli and E. Casiraghi, "Lung field segmentation in digital postero-anterior chest radiographs," in International Conference on Pattern Recognition and Image Analysis. Springer, 2005, pp. 736-745.

9. R. Sousa et al, "Comparative performance analysis of machine learning classifiers and dimensionality reduction algorithms in detection of childhood pneumonia", Procedia Computer Science, vol. 18, pp. 2579-2582, 2013.

10. Laurens van der Maaten, Geoffrey Hinton "Visualizing Data using t-SNE" 2008. 